\title{
Suppression of autoimmune disease after vaccination with autoreactive T cells that express Qa-1 peptide complexes
}

\author{
Vily Panoutsakopoulou, ${ }^{1,2}$ Katharina M. Huster, ${ }^{1,2}$ Nami McCarty,1,2 Evan Feinberg,1 Rijian Wang,,1,2 \\ Kai W. Wucherpfennig, ${ }^{1,3}$ and Harvey Cantor ${ }^{1,2}$
}

\begin{abstract}
1Department of Cancer Immunology \& AIDS, Dana-Farber Cancer Institute, Boston, Massachusetts, USA. 2Department of Pathology and 3Department of Neurology, Harvard Medical School, Boston, Massachusetts, USA.
\end{abstract}

\begin{abstract}
The ability of autoreactive $T$ cells to provoke autoimmune disease is well documented. The finding that immunization with attenuated autoreactive $T$ cells ( $T$ cell vaccination, or TCV) can induce $T$ cell-dependent inhibition of autoimmune responses has opened the possibility that regulatory $T$ cells may be harnessed to inhibit autoimmune disease. Progress in the clinical application of TCV, however, has been slow, in part because the underlying mechanism has remained clouded in uncertainty. We have investigated the molecular basis of TCVinduced disease resistance in two murine models of autoimmunity: herpes simplex virus-1 (KOS strain)-induced herpes stromal keratitis and murine autoimmune diabetes in non-obese diabetic (NOD) mice. We find that the therapeutic effects of TCV depend on activation of suppressive CD8 cells that specifically recognize Qa-1-bound peptides expressed by autoreactive CD4 cells. We clarify the molecular interaction between Qa-1 and self peptides that generates biologically active ligands capable of both inducing suppressive CD8 cells and targeting them to autoreactive CD4 cells. These studies suggest that vaccination with peptide-pulsed cells bearing the human equivalent of murine Qa-1 (HLA-E) may represent a convenient and effective clinical approach to cellular therapy of autoimmune disease.
\end{abstract}

\section{Introduction}

Autoreactive $\mathrm{T}$ cells are part of the normal $\mathrm{T}$ cell repertoire $(1,2)$ and can provoke autoimmune disease after abnormal environmental stimuli, as documented by adoptive transfer of autoreactive $\mathrm{T}$ cells in murine models of diseases $(3,4)$. The cellular mechanisms that may inhibit this process are not well understood. The suggestion that regulatory $\mathrm{T}$ cells might be harnessed to inhibit disease came from findings that immunization with attenuated autoreactive $\mathrm{T}$ cells conferred resistance to subsequent disease induction (3, $5)$. This procedure, termed $\mathrm{T}$ cell vaccination (TCV) (6), has been examined in several animal models of tissue-specific autoimmune diseases $(6,7)$ and in small studies of MS patients $(8,9)$. Progress in the clinical application of TCV, however, has been slowed, in part, because the underlying mechanism of TCV is not well understood.

Recent studies have suggested that the protective effect of TCV may reflect induction of regulatory CD8 cells (10-16). For example, depletion of CD8 cells prior to TCV abrogates its protective effect (12) and $\mathrm{T}_{\mathrm{S}}$ activity following immunization with lymphocytes is mediated by CD8 cells (17). The mechanism(s) that lead to induction of suppressive CD8 cells remains unclear, however. Although autoreactive T cell receptors (TCRs) have been implicated in the genesis of immunoregulatory cells, the molecular interactions that underlie presentation of autoreactive TCR to the immune system in a way that induces CD8-dependent suppression have not been clarified.

Nonstandard abbreviations used: concanavalin A (conA); experimental

autoimmune encephalomyelitis (EAE); herpes simplex virus 1 (HSV-1); herpes stromal keratitis (HSK); myelin basic protein (MBP); non-obese diabetic (NOD); T cell receptor (TCR); T cell vaccination (TCV).

Conflict of interest: The authors have declared that no conflict of interest exists.

Citation for this article: J. Clin. Invest. 113:1218-1224 (2004).

doi:10.1172/JCI200420772.
Attempts at vaccinating using isolated TCR peptides have not met with uniform success, and the effects of blocking Ab's against classical MHC class-Ia molecules expressed by autoreactive CD4 cells used for TCV have been inconclusive. Lysis of autologous CD4 cells by CD8 cells from vaccinated MS patients has been reported to be prevented by class Ia $\mathrm{Ab}(8,13)$, while other studies indicate that inhibitory interactions between CD8 cells and $\mathrm{V}_{\beta} 8^{+}$murine CD4 clones is blocked by Ab to the class Ib molecule Qa-1 (17). Although the in vitro responses of CD8 cells is restricted by Qa- 1 expressed on target cells $(10,17)$, the contribution of Qa-1 peptide complexes to the development of inhibitory activity after TCV is not clear.

We have analyzed the effects of TCV in two murine models of autoimmune disease: herpes stromal keratitis (HSK), which is a clinical consequence of corneal infection by herpes simplex virus 1 (HSV-1) and represents a leading cause of human blindness (18), and non-obese diabetic (NOD) mice, a murine model of type 1 diabetes. We show that the therapeutic effects of TCV depend on induction of suppressive CD8 cells that specifically recognize Qa-1 peptide complexes expressed by autoreactive CD4 cells in two murine models of autoimmune disease. These studies provide new insight into the molecular basis of this inhibitory $\mathrm{T}$ cell- $\mathrm{T}$ cell interaction that is mediated by TCV and suggest new approaches to this cellular therapy.

\section{Methods}

Mice. Six- to eight-week-old female C.AL-20 (BALB/c-Igh), $\mathrm{BALB} / \mathrm{c}, \mathrm{BALB} / \mathrm{c}-I F N-\gamma^{-1}, \mathrm{BALB} / \mathrm{c}-I L-10^{-/-} \mathrm{BALB} / \mathrm{c}-R A G-2^{-1-}$, C.B17-SCID, NOD, $129 \mathrm{~Sv}$, and $129 \mathrm{SvIFN-} \gamma R^{-/-}$mice were purchased from The Jackson Laboratory (Bar Harbor, Maine, USA). All mice were housed in microisolator cages in the Dana-Farber Animal Facility and, when appropriate, in biosafety level 2. In all 
the experiments each group represents at least eight mice, and each experiment was performed at least twice.

Ocular infections and scoring of HSK. Mice were infected with $4 \times 10^{5}$ PFU of HSV-1 (KOS strain) in the right eye, and the extent of the disease was scored on different days within 14-15 days after infection as described (19). Disease score is summarized as the HSK index, which equals severity (mean clinical score) multiplied by percentage of incidence multiplied by ten, where severity of clinical stromal keratitis was quantified based on the degree of corneal opacity: 1 , less than or equal to $25 \%$ of cornea; 2 , less than or equal to $50 \%$; 3 , less than or equal to $75 \%$; $4,75-100 \%$. (For all disease severity data points SD was $<0.5$.) Incidence was the percentage of mice with a severity score greater than or equal to 1 .

$\mathrm{CD}^{+} \mathrm{TCV}$ protocol. $\mathrm{CD} 4^{+} \mathrm{T}$ cells were purified from the right cervical draining LNs of C.AL-20 mice 10 days after infection of the right eye with $4 \times 10^{5}$ PFU HSV-1 (KOS) by magnetic negative selection (Dynabeads; Dynal Inc., Lake Success, New York, USA) using antiCD8 (53-6.7), anti-B220 (RA3-6B2), anti-Gr-1 (RB6-8C5), and anti-Mac-1 (M1/70) mAb's, and cultured in the presence of concanavalin A (conA) $(5 \mu \mathrm{g} / \mathrm{ml})$ for 40 hours. The $\mathrm{CD}^{+} \mathrm{T}$ cells (approximately $97 \%$ pure $\mathrm{CD}^{+}$cells as confirmed by FACS analysis) were then washed three times with RPMI medium, irradiated at $30 \mathrm{~Gy}$, and resuspended at $1.25 \times 10^{7}$ cells $/ \mathrm{ml}$ in RPMI. Mice were injected in the tail vein with $200 \mu \mathrm{l}$ of the cell suspension $\left(2.5 \times 10^{6}\right.$ cells/mouse), and 3 weeks later were ocularly infected with HSV-1 (KOS). In some experiments cells from the C1-6 CD4 ${ }^{+} \mathrm{T}$ cell clone reacting to $\gamma 2 \mathrm{a}^{\mathrm{b}}$ peptide and cross-reacting to a corneal antigen were used instead. For some experiments, cells from the $\mathrm{O} 3 \mathrm{CD}^{+}{ }^{+} \mathrm{T}$ cell line or the DO11.10 TCR transgenic mice that react to OVA were used as controls. In experiments where sorted $\mathrm{CD}^{+}$cells were used, $10^{6}$ purified $\mathrm{CD}^{+} \mathrm{T}$ cells of draining LNs of ocularly HSV-1infected mice were injected per mouse. For TCV experiments in NOD mice pancreatic LNs from 15-week-old female NOD donors were obtained during the insulitic stage of disease in NOD mice and were treated with conA for 40 hours followed by irradiation (30 Gy) before i.v. injection $\left(2.5 \times 10^{6}\right.$ cells/mouse $)$ into 11 -week-old female NOD recipients. Vaccinated animals were observed for onset of diabetes; disease progression was measured by serum (or urine, where indicated) glucose levels and mortality. Cell preparations and purifications were performed in the same way as in TCV for HSK.

In blocking experiments, cells were incubated with a mouse IgG-1 Ab that binds to Qa- $1^{\mathrm{b}}$ (anti-Qa-1 ${ }^{\text {b }}$ was kindly provided by M. Soloski, Johns Hopkins University, Baltimore, Maryland, USA), or isotype-matched control Ab (IgGK; A112-2), or anti-MHC class I Ab (H-2D ${ }^{\text {b }}$ 34-2-12) for 1 hour at $4^{\circ} \mathrm{C}$ after 40 hours of in vitro stimulation with con $\mathrm{A}(5 \mu \mathrm{g} / \mathrm{ml})$ and prior to irradiation and injection into mice.

In some experiments, $\mathrm{O} 3$ cells were used for TCV after incubation with peptides $V_{\beta} 8.1$ (LLSWVALFL) or L9D (LLSWVALFD) (New England Peptide Inc., Gardner, Massachusetts, USA) as follows: $\mathrm{O} 3$ cells were stimulated for

\section{Figure 1}

40 hours at $37^{\circ} \mathrm{C}$ in RPMI $/ 10 \%$ FCS with conA $(5 \mu \mathrm{g} / \mathrm{ml})$ before addition of $\mathrm{V}_{\beta} 8.1$ peptide or L9D peptide (final concentration 0.5 $\mu \mathrm{g} / \mathrm{ml}$ ) for 1 hour. After cells were washed three times with RPMI and irradiated ( $30 \mathrm{~Gy}$ ), $2 \times 10^{6}$ cells (in $200 \mu \mathrm{l}$ RPMI) were injected per C.AL-20 mouse. In one experiment the peptides were dissolved in $25 \mu \mathrm{l} \mathrm{CFA}$ per mouse and subcutaneously injected into the back of anesthetized C.AL-20 mice that were 3 weeks later ocularly infected with HSV-1 (KOS) and scored for HSK.

Transfer experiments. For transfer of CD8 cells, these cells were purified from LNs and spleens 2 weeks after TCV by negative magnetic selection (Dynabeads; Dynal Inc.) with anti-CD4 (GK1.5), anti-B220 (RA3-6B2), anti-Gr-1 (RB6-8C5), and anti-Mac-1 (M1/70) mAb's. CD8 cells $\left(5 \times 10^{6} /\right.$ mouse) in $200 \mu 1$ PBS ( $97 \%$ pure CD8 as confirmed by FACS) were injected in the tail vein of recipient animals.

In some experiments CD8 cells from previously vaccinated WT, $I L-10^{-/-}$, and $I F N-\gamma^{-/}$mice were adoptively transferred into recipient CB.17-SCID mice together with CD8-depleted LN cells from WT BALB/c mice. For the experiments where T cells are transferred into $R A G-2^{-/-}$recipients 6 days before analysis, $2 \times 10^{6}$ cells from donors vaccinated with $\mathrm{V}_{\beta} 6^{+}$or $\mathrm{V}_{\beta} 8^{+} \mathrm{CD} 4$ cells were admixed with $10^{4}$ conA-activated $\mathrm{V}_{\beta} 6^{+}$or $\mathrm{V}_{\beta} 8^{+} \mathrm{CD} 4$ cells as indicated.

In vivo depletions. Groups of mice were depleted of CD8 cells prior to TCV and ocular infection by intraperitoneal injections (six times) with monoclonal anti-CD8 Ab $(2.43 ; 25 \mu \mathrm{g} /$ dose). The depletion (97\%) was confirmed by FACS analysis.
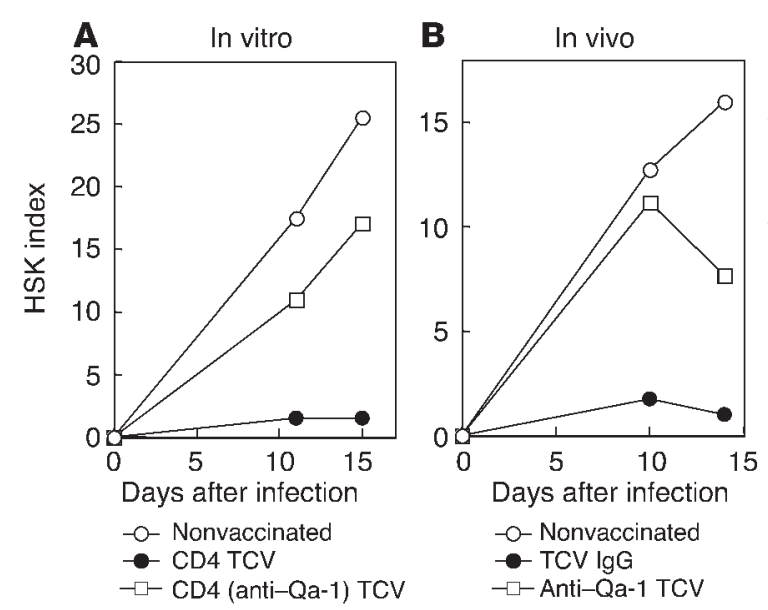

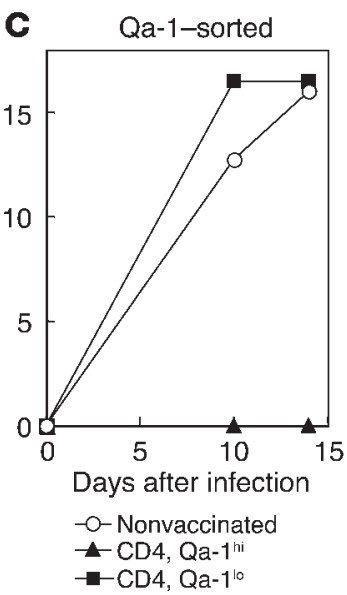

Qa-1 $1^{\mathrm{b}}$ expression on CD4 cell vaccine is essential for protection. Effects of monoclonal anti-Qa-1 Ab on TCV in vitro (A) and in vivo (B). (A) C.AL-20 mice were vaccinated with CD4 cells isolated from HSV-1-infected C.AL-20 mice. A group of mice was vaccinated with the same CD4 cells but incubated with blocking anti-Qa-1 mAb (open squares) or isotype control (filled circles) or were nonvaccinated controls (open circles) as described in Methods. Two weeks later, vaccinated mice were ocularly infected with HSV-1 (KOS) and scored for HSK. Each group consisted of ten mice, and results are representative of three independent experiments. (B) C.AL-20 mice were intraperitoneally injected with anti-Qa-1 mAb (10 $\mu \mathrm{g} / \mathrm{mouse})$ at the time of TCV (open squares) or isotype control (filled circles) or were nonvaccinated controls (open circles). Two weeks later, vaccinated mice were ocularly infected with HSV-1 (KOS) and scored for HSK. Each group consisted of 10-20 mice, and results are representative of three independent experiments. (C) Qa-1 sorting. C.AL-20 mice were vaccinated with CD4 cells isolated from HSV-1-infected C.AL-20 mice. These CD4 cells were sorted according to their level of Qa-1 surface expression (high, filled triangles, versus low, filled squares) or nonvaccinated controls (open circles). Both groups were ocularly infected with HSV-1 (KOS) and scored for HSK as described in Methods. Each group consisted of 10-20 mice. Data are representative of two independent experiments. 


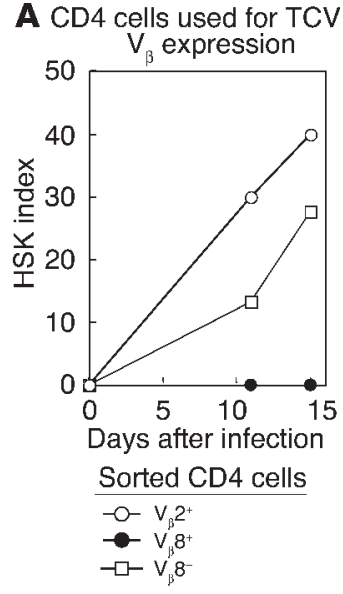

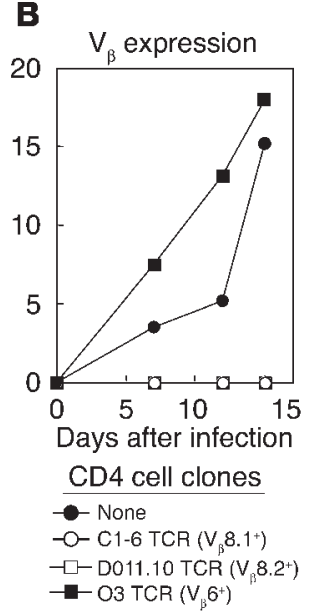
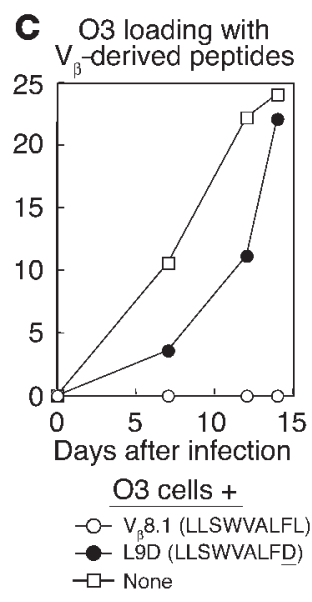

\section{Figure 2}

$\mathrm{V}_{\beta}$ expression and TCV. (A) TCR $\mathrm{V}_{\beta} 8^{+}$CD4 cells protect from development of HSK. C.AL-20 mice were vaccinated with CD4 cells (as described in Methods) sorted according to $T C R-V_{\beta}$ expression $\left(V_{\beta} 8^{+}\right.$, filled circles; $V_{\beta} 8^{-}$, open squares; $V_{\beta} 2^{+}$, open circles). All mice were ocularly infected with HSV-1 (KOS) and scored for HSK. Each group consisted of ten mice, and results are representative of three independent experiments. (B) BALB/c mice were vaccinated with cells of either a TCR $V_{\beta} 8^{+} C D 4$ $T$ cell clone reactive to a corneal antigen (C1-6, open circles), or a CD4 T cell clone reactive to an OVA-derived peptide (DO11.10; open squares), or a TCR $\mathrm{V}_{\beta} 6^{+} \mathrm{CD} 4 \mathrm{~T}$ cell clone reactive to an OVA-derived peptide (O3; filled square), or were unvaccinated controls (filled circle). All mice were ocularly infected with HSV-1 (KOS) and scored for HSK. Each group consisted of ten mice, and results are representative of four independent experiments. (C) Binding of a TCR-derived $\mathrm{V}_{\beta} 8.1$ peptide to Qa- 1 renders $\mathrm{O} 3$ cells protective. C.AL-20 mice were vaccinated with pure cells of the TCR $V_{\beta} 6^{+}$CD4 $\mathrm{T}$ cell clone $\mathrm{O} 3$ (open squares) or $\mathrm{O} 3$ cells incubated with a TCR-derived $\mathrm{V}_{\beta} 8.1$ peptide (open circles) or the mutated peptide L9D (filled circles). Two weeks later, all mice were ocularly infected with HSV-1 (KOS) and scored for HSK. Each group consisted of ten mice, and results are representative of three independent experiments.

Flow cytometry. Flow-cytometric analysis was performed to measure the Qa- $1^{\mathrm{b}}$ expression on the surface of the CD4 cells using a Coulter EPICS XL flow cytometer. LN cells or, in sorting experiments, purified CD4 cells were stained with anti-CD4 phycoerythrin-conjugated (PE-conjugated) Ab (PharMingen, San Diego, California, USA) and with an unconjugated primary Ab to Qa- $1^{\text {b }}$ followed by an anti-mouse IgG-1-FITC-conjugated $\mathrm{mAb}$ (PharMingen). CD4 cells were measured with a fluorescence-activated cell sorter (FACS) for their Qa-1 expression or sorted according to the level of $\mathrm{Qa}-1^{\mathrm{b}}$ expression on their surface.

Qa-1 tetramers. Qa- $1^{\mathrm{a}}$ and $\beta_{2}$-microglobulin were produced as inclusion bodies in Escherichia coli BL21 (DE3) carrying either the pET-23a/ $\mathrm{Q} a$ or the $\beta_{2}$-microglobulin plasmid. The inclusion bodies were purified and dissolved in urea buffer. The monomeric Qa-1 ${ }^{\mathrm{a}}$-peptide complexes were formed by combining the Qa- $1, \beta_{2}$-microglobulin, and the peptide in an arginine-folding buffer. The complexes were then purified on a Superdex-200 gel-filtration column (Pharmacia

\section{Figure 3}

TCV protects NOD mice from disease. Female NOD mice (11 weeks old) were vaccinated (filled circles) as described in Methods and observed for onset and progress of diabetes (left), determined by glucose levels in urine, and mortality (right). Unvaccinated controls are represented by open circles. Each group consisted of approximately 50 mice. Results are representative of more than seven independent experiments.
Biotech Inc., Piscataway, New Jersey, USA) before biotinylation with the BirA enzyme (Avidity, Denver, Colorado, USA). The biotinylated monomeric complexes were purified again with Superdex-200 gel-filtration column. Streptavidin-PE (Molecular Probes Inc., Eugene, Oregon, USA) was added to form the tetrameric reagent in a $4: 1$ ratio. The tetramer efficiency was measured by HPLC.

All experiments involving animals were performed in compliance with federal laws and institutional guidelines and have been approved by the Dana-Farber Cancer Institute Animal Care and Use Committee.

\section{Results}

\section{Autoreactive $\mathrm{CD}^{+}$cells protect from development of HSK when used as a vaccine}

Ocular infection of C.AL-20 or BALB/c mice with HSV-1 (KOS) triggers HSK, an autoimmune disease initiated by CD 4 cells that induce corneal destruction and blindness $(19,20)$. To test the effects of TCV on HSK, mice were vaccinated with purified CD4 cells from the LNs draining the eyes of mice that had developed HSK 10 days after HSV-1 (KOS) infection and then were irradiated after stimulation in vitro with conA (as described in Methods). Corneal inoculation of HSV-1 3 weeks after intravenous TCV revealed that vaccinated mice developed a mild and transient HSK (mean index = 2.5); nonvaccinated control mice developed severe disease (mean index $=25$ ) by day 15 . Vaccination-induced protection was durable: none of the vaccinated mice developed HSK (100\% protection) after a second ocular infection with HSV-1 (KOS). Vaccination was also specific: irradiated mice inoculated with conA-stimulated CD4 cells from uninfected C.AL-20 mice were not protected from HSK (data not shown and see below).

To determine whether the protective effects of TCV depend on expression of the class Ib molecule Qa- 1 by activated CD 4 cells we examined the effects of monoclonal anti-Qa- $1^{\mathrm{b}} \mathrm{Ab}$ on protection. Vaccination using cells that had been incubated with anti-Qa- $1^{\text {b }}$ $\mathrm{Ab}$ failed to efficiently inhibit disease compared with CD4 cells incubated with an isotype-matched control $\mathrm{Ab}$, which induced strong disease resistance (Figure 1A). Injection of monoclonal anti-Qa-1 blocking Ab in vivo along with TCV also largely abrogated its suppressive effects (Figure 1B). Finally, vaccination with FACS-sorted Qa-1 ${ }^{\text {hi }}$ CD4 cells (mean fluorescence intensity greater than 100$)$ yielded complete $(100 \%)$ protection, while vaccination with Qa-1 ${ }^{\text {lo }}$ CD4 cells (mean fluorescence intensity less than 40)

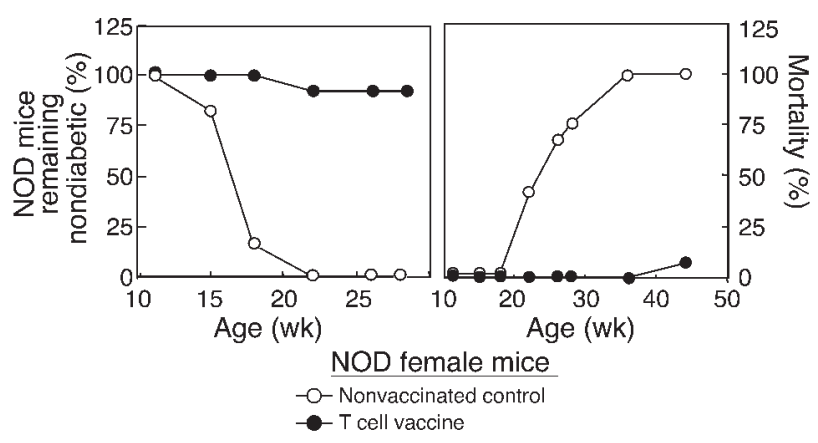


Table 1

TCV-induced protection of DM in NOD mice

\begin{tabular}{lccccccccc}
\hline & \multicolumn{10}{c}{ Source of LN T cells } \\
& \multicolumn{10}{c}{ Nonvaccinated } & \multicolumn{1}{c}{ Mesenteric } & \multicolumn{2}{c}{ Cervical } & \multicolumn{2}{c}{ Pancreatic T cells } & Pancreatic CD4 cells \\
NOD (female) mice & $17 \mathrm{wk}$ & $30 \mathrm{wk}$ & $17 \mathrm{wk}$ & $30 \mathrm{wk}$ & $17 \mathrm{wk}$ & $30 \mathrm{wk}$ & $17 \mathrm{wk}$ & $30 \mathrm{wk}$ & $20 \mathrm{wk}$ \\
Mortality & $50 \%$ & $90 \%$ & $0 \%$ & $80 \%$ & $25 \%$ & $75 \%$ & $0 \%$ & $0 \%$ & $0 \%$ \\
Elevated glucose & $75 \%$ & $100 \%$ & $60 \%$ & $100 \%$ & $60 \%$ & $100 \%$ & $0 \%$ & $10 \%$ & $30 \%$ \\
\hline
\end{tabular}

NOD female mice (11 weeks old) were vaccinated with T or CD4 cells as described in Methods. Each experimental group consisted of $40-90$ mice.

did not exert detectable disease protection (Figure 1C). Both Qa-1 hi and Qa- $1^{\text {lo }} \mathrm{CD} 4$ subpopulations were equally activated according to expression of activation-associated surface markers CD44 and CD62, as judged by forward light scatter upon FACS analysis (not shown). Possibly, Qa-1 ${ }^{\text {hi }}$ cells may represent a subpopulation of activated CD4 cells that displays relatively high levels of non-Qdm peptides, including those derived from the TCR. An additional property of these cells that may be relevant to their immunogenicity is the increased levels of surface TCR (but not other surface receptors, such as CD4; data not shown).

\section{$T C V$-induced protection depends on TCR Vb peptide-Qa-1 complexes expressed by $\mathrm{CD} 4$ cells}

The autoreactive C1-6 TCR $\left(\mathrm{V}_{\beta} 8.1 \mathrm{~V}_{\alpha} 11\right)$ that confers HSK has been defined in polyclonal populations and tested in mice that carry the C1-6 TCR transgene (21). Vaccination using purified $\mathrm{V}_{\beta} 8^{+} \mathrm{CD} 4$ cells from normal $(\mathrm{BALB} / \mathrm{c})$ mice resulted in $100 \%$ protection from HSK, while vaccination using $V_{\beta} 8$-depleted $C D 4$ cells or purified $\mathrm{V}_{\beta} 2^{+} \mathrm{CD} 4$ cells did not protect from HSK (Figure $2 \mathrm{~A}$ ). We compared the protective effects of three different BALB/c $\mathrm{V}_{\beta}{ }^{+}$TCRs: $\mathrm{V}_{\beta} 8.1^{+} /$class II ${ }^{\mathrm{d}}$-restricted C1-6 TCR $(20,21)$; DO11.10, a $\mathrm{V}_{\beta} 8.2^{+}$ TCR specific for an OVA-derived peptide/class II ${ }^{d}(22)$; and O3, a $\mathrm{V}_{\beta} 6^{+}$TCR specific for a second OVA-derived peptide/class II ${ }^{\mathrm{d}}(23)$. Vaccination with activated, irradiated $V_{\beta} 8.2^{+}$DO11.10 cells and $\mathrm{V}_{\beta} 8.1^{+} \mathrm{C} 1-6$ cells successfully inhibited HSK, while vaccination with $\mathrm{V}_{\beta} 6^{+} \mathrm{O} 3$ cells did not (Figure $2 \mathrm{~B}$ ).

\section{$T C V$-induced protection depends on $V_{\beta} 8$ peptide binding to $Q a-1^{b}$}

These results suggested that the TCV ligand comprised a $V_{\beta} 8$ derived peptide bound to $\mathrm{Qa}-1^{\mathrm{b}}$ on activated CD4 cells. Analysis of $V_{\beta} 8.1$ revealed a single motif (LLSWVALFL) that might allow refolding of purified Qa-1 ${ }^{\text {b }}$ (but not MHC class I molecules). Qa-1folding activity with this peptide was confirmed according to HPLC analysis of $V_{\beta} 8$ peptide: Qa-1 complexes and tetramers (24). This finding allowed a direct test of the role of this $V_{\beta^{-}}$ Qa-1 ligand in TCV: Did loading of $\mathrm{V}_{\beta} 6^{+} \mathrm{O} 3$ cells with the Qa-1binding, $\mathrm{V}_{\beta} 8$-derived peptide (which were themselves inactive; Figure $2 \mathrm{~B}$ ) confer protective activity on this clone? We found that vaccination with activated irradiated $V_{\beta} 6^{+} \mathrm{CD} 4$ cells incubated with $V_{\beta} 8.1$ peptide resulted in $100 \%$ protection (Figure $2 \mathrm{C}$ ), while vaccination with $\mathrm{V}_{\beta} 6^{+} \mathrm{CD} 4$ cells incubated with a peptide homologue containing a $\mathrm{L} \rightarrow \mathrm{D}$ amino acid exchange (LLSWVALFD) that abolished peptide-induced folding of Qa-1 had no protective effect (Figure $2 \mathrm{C}$ ). The possibility that the $\mathrm{V}_{\beta} 8.1$ peptide itself might confer protection independent of its interaction with Qa-1 was tested by injection of $V_{\beta} 8.1$ peptide admixed with Qa-1- O3 cells or CFA; these stimuli had no effect on HSK (data not shown).

\section{TCV-based inbibition of diabetes in NOD mice}

We asked whether vaccination with pancreatic LN T cells obtained during the insulitis stage of disease in NOD mice might preempt subsequent IDDM. This was the case, as measured by disease progression (serum glucose) and mortality (Figure 3). Pathogenic isletreactive $T$ cells are enriched in draining pancreatic LNs, particularly early in disease in NOD mice (25). NOD mice vaccinated with CD4 cells from pancreatic LNs but not inguinal or mesenteric LNs are protected from disease progression (Table 1).

\section{$T C V$-associated induction of suppressive CD8 cells}

The CD8 cell response is essential for TCV-induced protection against HSK because CD8 depletion abolished the protective effect of TCV on HSK (Figure 4A), and purified CD8 cells from donors vaccinated
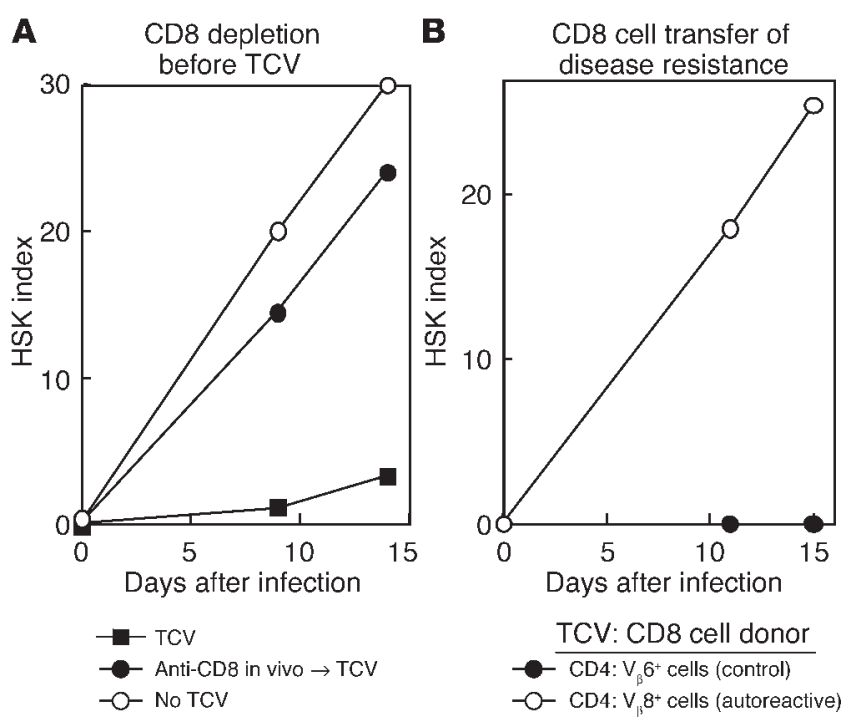

Figure 4

TCV induces CD8 ${ }^{+}$T suppressor cells: analysis in HSK. (A) CD8 depletion before TCV. Depletion of CD8 T cells abolishes TCV-induced protection. C.AL-20 mice were depleted of CD8 T cells by intraperitoneal injection of depleting anti-CD8 Ab (filled circles) or isotype control (filled squares) prior to TCV and ocular infection with HSV-1. A group of mice was not vaccinated for comparison (open circles). Each group consisted of ten mice. and results are representative of three independent experiments. (B) CD8 cell transfer of disease resistance. Protection can be transferred by CD8 cell transfer from vaccinated mice. C.AL-20 mice received $\mathrm{CD} 8$ cells from donors vaccinated with either keratogenic $\mathrm{V} \beta 8^{+}$ C1- 6 cells (filled circles) or OVA-specific $\mathrm{V}_{\beta} 6^{+} \mathrm{O} 3$ cells (open circles). The recipients were ocularly infected with HSV-1 (KOS) and scored for HSK. Each group consisted of ten mice, and results are representative of three independent experiments. 


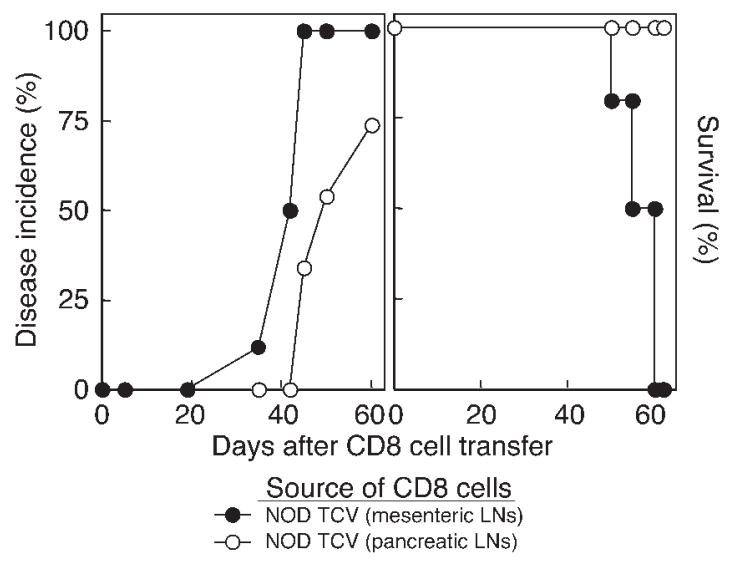

Figure 5

CD8 cells from vaccinated NOD mice inhibit disease progression. Female NOD mice that had received i.v. injection of $5 \times 10^{6} \mathrm{CD} 8$ cells from donors vaccinated with either $T$ cells from pancreatic (open circles) or mesenteric LNs (filled circles) were observed for disease incidence (glucose $>300 \mathrm{mg} / \mathrm{dl}$ ) and survival.

with $\mathrm{V}_{\beta} 8^{+}$but not $\mathrm{V}_{\beta} 6^{+}$cells inhibited disease in syngeneic recipients (Figure 4B). Analysis of the effects of TCV in NOD mice revealed that TCV-based disease inhibition also reflected TCV-based induction of host CD8 cells, similar to findings in HSK. CD8 cells from NOD mice vaccinated with CD4 cells from draining pancreatic (but not inguinal or mesenteric LNs) inhibited IDDM in NOD recipients, according to both serum glucose and survival (Figure 3). CD8 cells from these vaccinated NOD donors also completely inhibited disease development after transfer into NOD.RAG-2-/- hosts that had received CD4 cells from 14-week-old female NOD donors (not shown).

\section{Analysis of inhibitory CD8-CD4 cell interactions in RAG-2-/- mice} The finding that the inhibitory effects of TCV were associated with the development of a population of CD8 cells that can transfer suppression (Figure 4 and Figure 5) suggest that suppression may be mediated by CD 8 cells that can recognize autoreactive pathogenic CD4 cells. Additional studies were carried out to more directly define this potential $V_{\beta}$-dependent inhibitory interaction between CD4 and CD8 cells in $R A G-2^{--}$hosts. We found that expansion of $\mathrm{V}_{\beta} 8^{+} \mathrm{CD} 4$ cells in $R A G-2^{-/-}$hosts was inhibited by CD8 cells from donors vaccinated with $\mathrm{V}_{\beta} 8^{+}$but not $\mathrm{V}_{\beta} 6^{+} \mathrm{CD} 4$ cells, as indicated by a $75 \%$ reduction of $\mathrm{V}_{\beta} 8^{+} \mathrm{C} 1-6 \mathrm{CD} 4$ cells at day 6 . Conversely, $\mathrm{CD} 8$ cells from donors vaccinated with $\mathrm{V}_{\beta} 6^{+} \mathrm{CD} 4$ cells inhibited target $\mathrm{V}_{\beta} 6^{+}$but not $\mathrm{V}_{\beta} 8^{+} \mathrm{CD} 4$ cells in RAG-2 ${ }^{-/-}$hosts (Figure 6).

\section{Discussion}

The precise mechanism underlying the effects of TCV has not been elucidated since 1981 when Cohen and colleagues coined the term to describe the administration of activated attenuated autoimmune $T$ cells (6). Although these and other studies have documented the inhibitory effects of TCV on the progression of autoimmune disease in experimental animal models, the underlying mechanism has remained poorly understood. As a result, approaches based on TCV have had varying degrees of success in clinical trials. The studies described here are aimed at a mechanistic understanding of TCV. They suggest that TCR $V_{\beta}$-derived peptides associated with Qa-1 (the murine homologue of HLA-E) on activated autoreactive CD4 cells can activate CD8-dependent suppression and inhibit autoimmunity.
We have analyzed TCV in the context of HSK and NOD disease because these models have several advantages over other murine models of autoimmunity. Since HSV-1 (KOS) infection of $\mathrm{BALB} / \mathrm{c}$ mice is routinely followed by a CD4-dependent autoimmune attack against corneal tissue, this model allowed analysis of TCV-induced protection against the autoimmune portion of an antiviral response after infection. We also analyzed the effects on the spontaneous development of diabetes in NOD mice. Both murine models allow testing of the efficacy of TCV on the development of autoimmunity in settings that more closely mimic the clinical development of autoimmunity in the absence of deliberate immunization with self peptide. In both cases, in vivo depletion of CD8 cells prior to TCV abolished its protective effects, prompting us to further probe the role of CD8 cells in regulating disease development. Transfer of disease protection into naive recipient mice by CD8 cells from donors that had received TCV delineated this subset as a central mediator of the inhibitory effects of TCV. The ability of CD8 cells from mice vaccinated with $\mathrm{V}_{\beta} 6^{+}$or $\mathrm{V}_{\beta} 8^{+} \mathrm{CD} 4$ cells to inhibit the expansion of CD4 cells in a $\mathrm{V}_{\beta}$-specific fashion indicated that a direct CD8-CD4 inhibitory interaction may be sufficient to mediate the disease-inhibitory effects of TCV. It remains possible that other (auxiliary) regulatory cells generated by TCV may also contribute to protection. Resistance to antigen-induced experimental autoimmune encephalomyelitis (EAE), for example, is also associated with regulatory $\mathrm{CD}^{+} \mathrm{T}$ cells that may recognize peptides from autoreactive TCR (26) and enhance generation of CD8 $\mathrm{T}_{\mathrm{S}}(27)$.

\section{Surface ligands on autoreactive $\mathrm{CD} 4$ cells induce regulatory CD8 cells}

EAE-inducing T cell lines specific for different components of myelin basic proteins (MBPs) did not prevent disease induced by different MBPs (28). In contrast, TCV against EAE cross-protected against autoimmune arthritis (29). These and other studies indicated that the protective effects of TCV do not depend on the antigen specificity of CD4 cells used for vaccination. Qa-1 can present a number of foreign (30) and self peptides (31). Although regulatory CD8 cells generated by TCV in MS patients did not recognize MBP-reactive CD4 clones bearing TCR chains that differed from the immunizing $\mathrm{CD}^{+}$clone, these $\mathrm{CD} 8$ clones recognized several distinct MBP-reactive $\mathrm{CD} 4$ clones that shared the same $\left(\mathrm{V}_{\alpha} 15\right)$ chain (14). Jiang et al. have demonstrated the important role of $\mathrm{V}_{\beta}$-specific anti-MBP clones in the induction of TCV-based protection: vaccination with

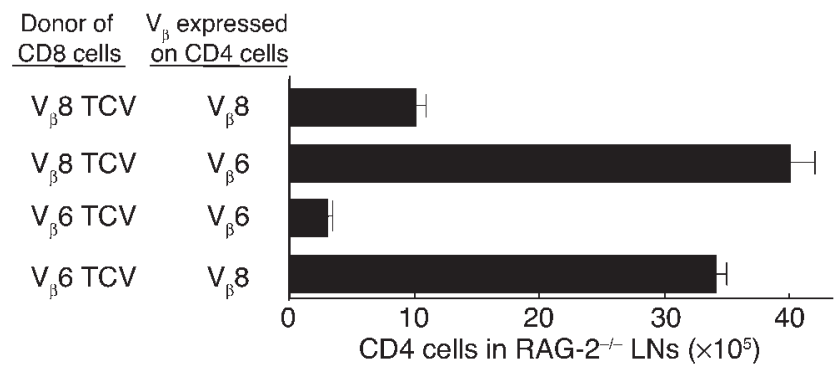

\section{Figure 6}

Interaction between CD4 and CD8 T cells in RAG-2-/- hosts. T cells transferred into BALB/c-RAG- $2^{-/-}$recipients 6 days before analysis. CD8 cells $\left(2 \times 10^{6}\right)$ from donors vaccinated with $V_{\beta} 6^{+}$or $V_{\beta} 8^{+} C D 4$ cells admixed with $10^{5}$ conA activated $\mathrm{V}_{\beta} 6^{+}$or $\mathrm{V}_{\beta} 8^{+} \mathrm{CD} 4$ cells as indicated. Results are representative of three independent experiments. 
three different $\mathrm{V}_{\beta} 8^{+} \mathrm{MBP}$-reactive $\mathrm{CD} 4 \mathrm{~T}$ cell clones protected against disease induced by another MBP-reactive $\mathrm{V}_{\beta} 8^{+} \mathrm{CD} 4 \mathrm{~T}$ cell clone, while $\mathrm{V}_{\beta} 6^{+} \mathrm{MBP}$-reactive $\mathrm{CD} 4 \mathrm{~T}$ cell clones did not (12).

Previous studies of HSK induced by HSV-1 (KOS) have established that pathogenic autoreactivity resides in a numerically small subpopulation of $\mathrm{CD} 4$ cells that express a $\mathrm{V}_{\beta} 8.1^{+} \mathrm{TCR}$ and crossreact with an HSV-1 viral epitope $(19,20)$. Vaccination with activated (polyclonal) $\mathrm{V}_{\beta} 8^{+} \mathrm{CD} 4^{+} \mathrm{T}$ cells from nonimmune mice conferred complete protection against HSK as did vaccination with $V_{\beta} 8.2^{+}$DO11.10 cells (reactive to an OVA peptide) and $V_{\beta} 8.1^{+}$C1-6 cells (see Figure 2B). Protection against HSK was not achieved after vaccination with conA-activated $\mathrm{CD}^{+} \mathrm{T}$ cells (containing the full spectrum of $V_{\beta}{ }^{+}$cells) or purified $V_{\beta} 2^{+}$or $V_{\beta} 6^{+}$cells. Both $V_{\beta} 8.1$ and $V_{\beta} 8.2$ share an identical Qa-1-binding motif (LLSWVALFL), which allows refolding of Qa-1- $\beta_{2} \mathrm{~m}$, but not MHC class I- $\beta_{2}$ m molecules in vitro (24). $\mathrm{A}_{\beta} 6^{+}$clone of $\mathrm{CD} 4$ cells that expresses high levels of Qa-1 after activation (but lacks a Qa-1-binding motif within the $\mathrm{V}_{\beta} 6$ protein) was programmed to induce HSK protection after pulsing with the LLSWVALFL peptide, but not an L9D mutant peptide (which does not allow refolding of Qa-1- $\beta_{2} \mathrm{~m}$; see Methods for incubation conditions). Since immunization with the $V_{\beta} 8$-derived peptide alone (in CFA) was not protective, these data indicate that cellular vaccination provides a peptide-Qa-1 complex that may specifically activate and expand Qa-1-restricted suppressive CD8 cells that can target autoreactive CD4 cells that express Qa-1-containing ligands. Although these results delineate the essential role of a $\mathrm{Q} a-1-\mathrm{V}_{\beta} 8$ peptide complex in TCV-induced resistance, they do not define the potential role(s) of other Qa-1-binding peptides, including naturally occurring Qdm and HSP-60. Although Qdm, HSP-60, and other Qa-1-peptide complexes are not sufficient for $\mathrm{TCV}$-based protection in the absence of the $\mathrm{V}_{\beta} 8$-Qa-1 ligand, they may, for example, regulate peptide exchange. We postulate that a shift in the proportions of the high-affinity Qdm-Qa-1 complex in favor of the lower-affinity HSP-60-Qa-1 complex 48 hours after activation of CD4 cells may facilitate exchange between exogenous peptides and the Qa-1 molecule (32-34).

Studies attempting to address the role of classical MHC class I molecules in TCV have yielded conflicting results $(8,13,35)$. Qa-1 was initially described as a surface molecule expressed on activated CD4 cells that interacted with suppressive CD8 cells (36-39), and antisera to Qa-1 but not class I blocked $\mathrm{CD}^{+}$killing of autoreactive $\mathrm{CD} 4^{+} \mathrm{T}$ cells in vitro (17). These observations may not fully reflect the situation in vivo, however. Since optimal protection was achieved after enrichment of CD4 cells that strongly express $\mathrm{Q} a-1^{\mathrm{b}}$ (see Figure 1C), while Ab blockade of Qa- $1^{\mathrm{b}}$ expression on irradiated activated CD4 cells used for TCV abolished protective activity, we concluded that $\mathrm{Qa}-\mathrm{1}^{\mathrm{b}}$ expression on $\mathrm{CD} 4$ cells is essential for successful TCV-based disease protection. We achieved optimal TCV efficacy using CD4 cells after conA-dependent stimulation in vitro for 40 hours; vaccination with nonactivated CD 4 cells was ineffective (40) as was vaccination with CD4 cells that had been stimulated for 24 or 72 hours (see Methods). A shift in the proportion of Qdm and HSP-60-containing complexes in favor of the latter 24-48 hours after activation of CD4 cells may facilitate exchange with LLSWVALFL (and other exogenous peptides), as discussed above, on activated T cells. An additional property of Qa-1hi cells that may be relevant to their immunogenicity entails increased levels of surface TCR (but not other surface receptors, such as CD4) displayed by these cells compared with the $\mathrm{Qa}-1^{10}$ cohort of activated $\mathrm{T}$ cells (data not shown).

\section{Regulatory sublineages of $\mathrm{CD} 4$ and $\mathrm{CD} 8$ cells}

Analysis of regulatory $\mathrm{T}$ cells in tissue-specific autoimmune disease has implicated a $\mathrm{CD} 25^{+}$subpopulation of $\mathrm{CD} 4$ cells in some models and IL-4-producing NK T cells in others (41). Previous studies of murine EAE, however, showed that TCV might result in induction of $\mathrm{CD}^{+} \mathrm{T}$ cells that lyse or damage autoreactive $\mathrm{CD} 4^{+} \mathrm{T}$ cells in vitro $(5,17)$. The tempo of this inhibitory response may be contrasted with naturally occurring $\mathrm{CD} 4 \mathrm{CD} 25^{+}$regulatory $\mathrm{T}$ cells that can interrupt expansion of self-reactive $\mathrm{T}$ cells. The development of Qa-1-restricted regulatory CD8 activity more closely resembles the development of CD8 CTL rather than regulatory CD4 cells. Once generated, CD8 CTL lyse virally infected target cells through TCRdependent recognition of viral peptides-class Ia MHC, while Qa-1restricted CD8 cells may eliminate a subpopulation of activated autoreactive $\mathrm{CD} 4$ cells through TCR-dependent recognition of self peptide-Qa-1 complexes $(42,43)$. In contrast, $C D 4 C D 25^{+}$regulatory activity generally acts at the early stages of primary responses (44, 45), either alone or through induction of regulatory CD8 cells (26).

Analysis of T cells expressing a TCR transgene specific for insulin associated with Qa-1 ${ }^{\mathrm{b}}$ has demonstrated that these cells are selected in the thymus through interactions with $\mathrm{Qa}-1 \mathrm{~b} /$ self peptides expressed primarily on hematopoietic cells. Both selection and antigen-recognition depend on expression of the TCR $\alpha / \beta$ and do not require NKG2 receptors (46). CD8 cells that recognize Qa-1 associated with the insulin peptide efficiently kill Qa-1-expressing lymphoblastoid target cells in the presence of intact soluble insulin, that is following transporter associated with antigen presentation-independent (TAP-independent) processing and presentation of insulin-derived peptide by Qa-1 on activated target cells (47). Possibly, Qa-1-restricted inhibitory activity of CD8 cells (Figure 6) may likewise reflect in vivo elimination of activated CD4 cells that display Qa-1-peptide complexes. Although the precise suppressor-effector mechanism remains to be determined, these data establish the central role of Qa-1-peptide complexes in the induction of TCV-based protection in two murine disease models and suggest potentially effective approaches to TCV in clinical settings, including the use of "universal" HLA-E ${ }^{+}$cell lines pulsed with target peptides as a potentially convenient and effective approach to immunosuppressive cellular therapy.

\section{Acknowledgments}

This work was supported in part by NIH research grants AI37562, AI13600, and AI48125 to H. Cantor. The authors wish to thank A. Sharpe for provision of DO11.10 transgenic mice; M. Soloski for anti-Qa-1 mAb; G. Doktor for lab assistance; and A. Angel for manuscript preparation and graphics.

Address correspondence to: Harvey Cantor, Dana-Farber Cancer Institute, SM 722, 44 Binney Street, Boston, Massachusetts 02115, USA. Phone: (617) 632-3348; Fax: (617) 632-4630; E-mail: Harvey_Cantor@dfci.harvard.edu.

Vily Panoutsakopoulou's present address is: Immunology Department, Transplantation Center, Foundation for Biomedical Research of the Academy of Athens, Athens, Greece.

Katharina M. Huster's present address is: Institute for Medical Microbiology, Immunology and Hygiene, Munich, Germany.

Vily Panoutsakopoulou and Katharina M. Huster contributed equally to this work. 
1. Cohen, I.R. 1992. The cognitive principle challenges clonal selection. Immunol. Today. 13:441-446.

2. Ota, K., et al. 1990. T-cell recognition of an immunodominant myelin basic protein epitope in multiple sclerosis. Nature. 346:183-187.

3. Holoshitz, J., Naparstek, Y., Ben-Nun, A., and Cohen, I.R. 1983. Lines of T lymphocytes induce or vaccinate against autoimmune arthritis. Science. 219:56-58.

4. Cantor, H. 2000. T-cell receptor crossreactivity and autoimmune disease. Adv. Immunol. 75:209-233.

5. Lider, O., Reshef, T., Beraud, E., Ben-Nun, A., and Cohen, I.R. 1988. Anti-idiotypic network induced by $\mathrm{T}$ cell vaccination against experimental autoimmune encephalomyelitis. Science. 239:181-183.

6. Ben-Nun, A., Wekerle, H., and Cohen, I.R. 1981. Vaccination against autoimmune encephalomyelitis with T-lymphocyte line cells reactive against myelin basic protein. Nature. 292:60-61.

7. Formby, B., and Shao, T. 1993 . T cell vaccination against autoimmune diabetes in nonobese diabetic mice. Ann. Clin. Lab. Sci. 23:137-147.

8. Correale, J., et al. 2000. T cell vaccination in secondary progressive multiple sclerosis. J. Neuroimmunol. 107:130-139.

9. Medaer, R., Stinissen, P., Truyen, L., Raus, J., and Zhang, J. 1995. Depletion of myelin-basic-protein autoreactive $T$ cells by T-cell vaccination: pilot trial in multiple sclerosis. Lancet. 346:807-808.

10. Noble, A., Zhao, Z.-S., and Cantor, H. 1998. Suppression of immune responses by CD8 cells: II. Qa-1 on activated B-cells stimulates CD8 cell suppression of Th2-dependent antibody responses. J. Immunol. 160:566-571.

11. Jiang, H., et al. 1995. Murine CD8+ T cells that specifically delete autologous CD4+ T cells expressing V $\beta 8$ TCR: role of the Qa-1 molecule. Immunity. 2:185-194.

12. Jiang, H., Braunstein, N.S., Yu, B., Winchester, R., and Chess, L. 2001. CD8+ T cells control the TH phenotype of MBP-reactive CD4+ T cells in EAE mice. Proc. Natl. Acad. Sci. U. S. A. 98:6301-6306.

13. Zhang, J., Medaer, R., Stinissen, P., Hafler, D., and Raus, J. 1993. MHC-restricted depletion of human myelin basic protein-reactive $\mathrm{T}$ cells by $\mathrm{T}$ cell vaccination. Science. 261:1451-1454.

14. Zhang, J., Vandevyver, C., Stinissen, P., and Raus, J. 1995. In vivo clonotypic regulation of human myelin basic protein-reactive $\mathrm{T}$ cells by $\mathrm{T}$ cell vaccination. J. Immunol. 155:5868-5877.

15. Zhang, Y.C.Q., Hong, J., Rivera, V.M., Killian, J., and Zhang, J.Z. 2000. Preferential recognition of TCR hypervariable regions by human anti-idiotypic $\mathrm{T}$ cells induced by $\mathrm{T}$ cell vaccination. J. Immunol. 164:4011-4017.

16. Gaur, A., Haspel, R., Mayer, J.P., and Fathman, C.G. 1993. Requirement for CD8+ cells in T cell receptor peptide-induced clonal unresponsiveness. Science. 259:91-94

17. Jiang, H., et al. 1998. T cell vaccination induces TCR $\mathrm{V} \beta$ specific, Qa-1 restricted regulatory CD8+ T cells. Proc. Natl. Acad. Sci. U. S. A. 95:4533-4537.

18. Dawson, C., and Togni, G. 1976. Herpes simplex eye infections: clinical manifestations, pathogenesis and management. Surv. Ophthalmol. 21:11-29.

19. Avery, A.C., et al. 1995. Resistance to herpes stromal keratitis conferred by an IgG2a-derived peptide. Nature. 376:431-434

20. Zhao, Z.-S., Granucci, F., Yeh, L., Schaffer, P.A., and Cantor, H. 1998. Molecular mimicry by herpes simplex virus-1: autoimmune disease after viral infection. Science. 279:1344-1347.

21. Panoutsakopoulou, V., et al. 2001. Analysis of the relationship between viral infection and autoimmune disease. Immunity. 15:137-147.

22. Chen, J., et al. 1993. Immunoglobulin gene rearrangement in B cell deficient mice generated by targeted deletion of the $\mathrm{JH}$ locus. Int. Immunol. 5:647-656

23. Friedman, S., Sillcocks, D., and Cantor, H. 1987. Alloreactivity of an OVA-specific T-cell clone. I. Stimulation by class II MHC and novel non-MHC B-cell determinants. Immunogenetics. 26:193-203.

24. Wang, R., Ramaswamy, S., Hu, D., and Cantor, H. 2001. Definition of a novel binding site on CD8 cells for a conserved region of the MHC class Ib molecule Qa-1 that regulates IFN-gamma expression. Eur. J. Immunol. 31:87-93.

25. Hoglund, P., et al. 1999. Initiation of autoimmune diabetes by developmentally regulated presentation of islet cell antigens in the pancreatic lymph nodes. J. Exp. Med. 189:331-339.

26. Kumar, V., and Sercarz, E.E. 1993. The involvement of $\mathrm{T}$ cell receptor peptide-specific regulatory CD4+ $\mathrm{T}$ cells in recovery from antigen-induced autoimmune disease. J. Exp. Med. 178:909-916.

27. Kumar, V., Stellrecht, K., and Sercarz, E. 1996. Inactivation of T cell receptor peptide-specific CD4 regulatory $\mathrm{T}$ cells induces chronic experimental autoimmune encephalomyelitis (EAE). J. Exp. Med. 184:1609-1617.

28. Holoshitz, J., Frenkel, A., Ben-Nun, A., and Cohen, I.R. 1983. Autoimmune encephalomyelitis (EAE) mediated or prevented by $\mathrm{T}$ lymphocyte lines directed against diverse antigenic determinants of myelin basic protein. Vaccination is determinant specific. J. Immunol. 131:2810-2813.

29. Lohse, A.W., Mor, F., Karin, N., and Cohen, I.R. 1989. Control of experimental autoimmune encephalomyelitis by $\mathrm{T}$ cells responding to activated T cells. Science. 244:820-822.

30. Lo, W.F., Ong, H., Metcalf, E.S., and Soloski, M.J. 1999. T cell responses to gram-negative intracellular bacterial pathogens: a role for CD8+ T cells in immunity to Salmonella infection and the involvement of MHC class Ib molecules. J. Immunol. 162:5398-5406

31. Kurepa, Z., Hasemann, C.A., and Forman, J. 1998 Qa-1b binds conserved class I leader peptides derived from several mammalian species. J. Exp. Med. 188:973-978.

32. Davies, A., et al. 2003. A peptide from heat shock protein 60 is the dominant peptide bound to Qa-1 in the absence of the MHC class Ia leader sequence peptide Qdm. J. Immunol. 170:5027-5033.
33. Gays, F., et al. 2001. Functional analysis of the molecular factors controlling Qa1-mediated protection of target cells from NK lysis. J. Immunol. 166:1601-1610

34. Brooks, A.G., et al. 1999. Specific recognition of HLA-E, but not classical, HLA class I molecules by soluble CD94/NKG2A and NK cells. J. Immunol. 162:305-313.

35. Sun, D., Qin, Y., Chluba, J., Epplen, J.T., and Wekerle, H. 1998. Suppression of experimentally induced autoimmune encephalomyelitis by cytolytic T-T cell interaction. Nature. 332:843-845.

36. Stanton, T.H., et al. 1978. The Qa-1 antigenic system. Relation of Qa-1 phenotypes to lymphocyte sets, mitogen responses and immune functions. J. Exp. Med. 148:963-973.

37. Cantor, H., et al. 1978. 2. Immunoregulatory circuits among T-cell subsets-2. Physiologic role of feedback inhibition in vivo: absence in NZB mice. J. Exp. Med. 147:1116-1125.

38. Cantor, H., et al. 1978. Immunoregulatory circuits among $\mathrm{T}$ cell sets. Identification of a subpopulation of T-helper cells that induces feedback inhibition. J. Exp. Med. 148:871-877.

39. Eardley, D.D., et al. 1978. Immunoregulatory circuits among T-cell sets. I. T-helper cells induce Tcell sets to exert feedback inhibition. J. Exp. Med. 147:1106-1115.

40. Naparstek, Y., et al. 1983. T lymphocyte lines producing or vaccinating against autoimmune encephalomyelitis (EAE). Functional activation induces peanut agglutinin receptors and accumulation in the brain and thymus of line cells. Eur. J. Immunol. 13:418-423.

41. Shevach, E.M. 2000. Regulatory T cells in autoimmunity. Annu. Rev. Immunol. 18:423-449.

42. Jiang, H., et al. 2003. Regulatory CD8+ T cells finetune the myelin basic protein-reactive $\mathrm{T}$ cell receptor $\mathrm{V}$ beta repertoire during experimental autoimmune encephalomyelitis. Proc. Natl. Acad. Sci.U.S. A. 100:8378-8383.

43. Jiang, H., and Chess, L. 2004. An integrated model of immunoregulation mediated by regulatory $\mathrm{T}$ cell subsets. Adv. Immunol. In press.

44. Sakaguchi, S., et al. 2001. Immunologic tolerance maintained by CD25+ CD4+ regulatory T cells: their common role in controlling autoimmunity, tumor immunity, and transplantation tolerance. Immunol. Rev. 182:18-32.

45. von Herrath, M.G., Dockter, J., and Oldstone, M.B. 1994. How virus induces a rapid or slow onset insulin-dependent diabetes mellitus in a transgenic model. Immunity. 1:231-242.

46. Sullivan, B.A., Kraj, P., Weber, D.A., Ignatowicz, L., and Jensen, P.E. 2002. Positive selection of a Qa-1restricted $\mathrm{T}$ cell receptor with specificity for insulin. Immunity. 17:95-105.

47. Tompkins, S.M., Kraft, J.R., Dao, C.T., Soloski, M.J., and Jensen, P.E. 1998. Transporters associated with antigen processing (TAP)-independent presentation of soluble insulin to alpha/beta $T$ cells by the class Ib gene product, Qa-1(b). J. Exp. Med. 188:961-971. 